\title{
CMS upgrade and future plans
}

\author{
Kerstin Hoepfner ${ }^{1}$, on behalf of the CMS collaboration \\ ${ }^{1}$ RWTH Aachen University, Phys.Inst. 3A, Otto-Blumenthal-Str., 52074 Aachen, Germany
}

\begin{abstract}
CMS plans for operation at the LHC phase-II unprecedented in terms of luminosity thus resulting in serious consequences for detector performance. To achieve the goal to maintain the present excellent performance of the CMS detector, several upgrades are necessary. To handle the high phase-II data rates, the readout and trigger systems are redesigned using recent technology developments. The high particle rates will accelerate detector aging and require replacement of the tracker and forward calorimeters. In addition, the muon system will be extended.
\end{abstract}

\section{The LHC phase-II conditions and detector consequences}

The CMS detector [1] was originally designed take data for about ten years at the LHC with a maximum instantaneous luminosity of $10^{34} \mathrm{~cm}^{-2} \mathrm{~s}^{-1}$, collecting up to $500 \mathrm{fb}^{-1}$ of total luminosity. Its main components are briefly summarized in the following. A solenoid magnet surrounds the inner tracker and calorimeters, providing a magnetic field of 3.8T . Silicon pixel and micro-stripsensors provide high resolution tracking and vertexing. Energies are measured with an electromagnetic (ECAL) $\mathrm{PbWO}_{4}$ crystal calorimeter along with a hadronic (HCAL) brass-scintillator sandwich calorimeter. The muon system, which is interleaved with the layers of the iron return yoke, exploits two complementary technologies: precision measurements are performed with drift-tube (DT) detectors in the barrel and cathode-strip chambers (CSC) in the endcaps, both are complemented with resistive plate chambers (RPC) for fast triggering and redundancy.

The planned high-luminosity (HL) operation during the LHC phase-II significantly exceeds the originally foreseen operating parameters, anticipating a five times larger instantaneous luminosity of up to $5 \times 10^{34} \mathrm{~cm}^{-2} \mathrm{~s}^{-1}$ and a total integrated luminosity of $3000 \mathrm{fb}^{-1}$. HL is essential to give access to rare processes and to enable precision measurements, as discussed in depth in other presentations at this conference. With $25 \mathrm{~ns}$ bunch spacing, this level of luminosity results in $\sim 140$ pile-up (PU) events on top of the hard-scattering signal interaction. Such conditions lead to serious consequences for the detector exceeding the original design:

1. Higher rates demand for increased trigger thresholds, unless dedicated measures such as increased granularity at trigger level are taken. 
2. Detector aging accelerates due to the high rate of charged particles (see Figure 1).

3. More bandwidth is needed to read out the data.

HL operation is foreseen to start around 2025 after a long shutdown (LS3) during which the necessary accelerator upgrades are to be performed. By 2025, the LHC detectors will have been operational for $\sim 20$ years, exceeding their foreseen lifetime by a factor of $\sim$ two. The installed components are based on technology from $\sim 30$ years ago and in particular electronics, computing and data transfer technologies have changed significantly since. It is thus mandatory to redesign electronics, trigger and DAQ to adapt to the HL conditions.

Particle rates increase with luminosity and are particularly high in the forward regions, as illustrated in Figure 1-right for the example of the muon system. The endcap rates more than double with respect to the barrel region. Most of the forward tracks are due to mediocre PU interactions and hence background, while the elusive physics processes of interest, e.g. vector-boson fusion, will also appear in this region and need to be extracted from the high background. Besides charged particles, many neutrons will be generated in the shielding, and beam-induced background affects the detectors near the beam pipe. From detailed simulations of particle interactions with the detector and shielding materials , the total particle flux (see Figure 1-left) is expected to reach up to $10^{7}-10^{8} \mathrm{~cm}^{-}{ }^{2} \mathrm{~s}^{-}$, with the maximum in the forward regions, which results in significant detector aging issues as they will be discussed in the following sections.
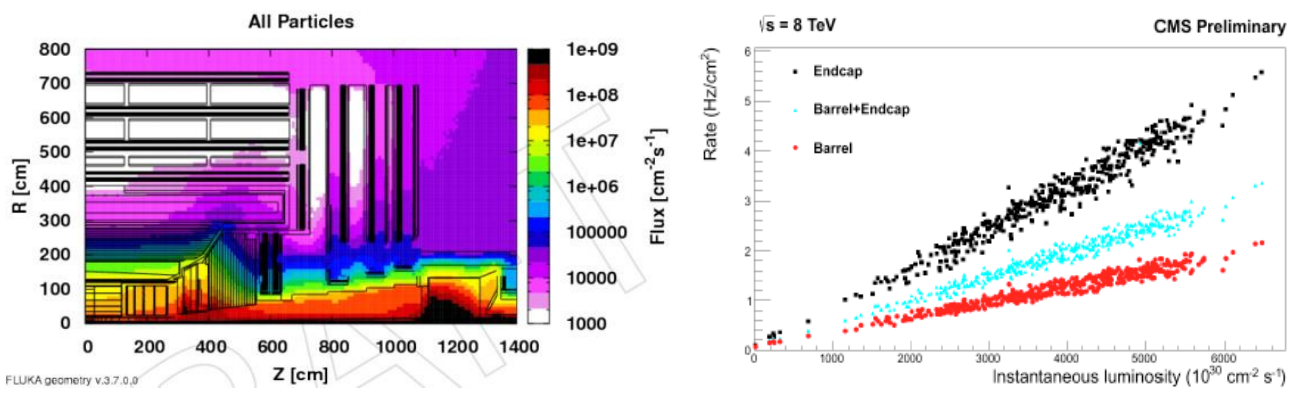

Figure 1. Radiation levels at the HL LHC, caused by PU and the resulting large number of charged tracks.

The present CMS level-1 trigger latency of $3.4 \mu \mathrm{s}$, dictated by the tracker readout, is planned to be increased to $12.5 \mu$ s to cope with the HL conditions. The present CMS level-1 trigger rate of $100 \mathrm{kHz}$ is determined the data acquisition rate, both reflecting the technologies prevalent $\sim 30$ years ago. Higher rates can be realized nowadays by means of high-bandwidth data-transfer link technologies from telecommunication industry. A trigger bandwidth of $750 \mathrm{kHz}$ will allow to operate a standard trigger menu designed for $200 \mathrm{PU}$ interactions taking into account a factor of 1.5 uncertainty on rate extrapolations. The implementation requires modification of readout electronics for subdetectors that will not be replaced during the upgrade, such as the ECAL barrel (see Section 3) and the muon system (see Section 4).

The trigger has to face the challenge to maintain the present trigger thresholds in a $5 \mathrm{x}$ higher PU. The turn-on curves need to be sharpened, and the rate of fake triggers must be reduced. Several detector modifications will be implemented for this purpose: ECAL crystals will be read out individually, thus matching the fine granularity of the new tracker (see Section 2) will permit noise (so-called spike) rejection and the use of timing information for PU mitigation. An additional muon detector (GE1/1) will enable a measurement of the bending angle in the very forward region, thus improving the 
accuracy of muon momentum measurement (see discussion in Section 4). The use of tracking information at the new level-1 tracking trigger (see discussion in Section 2) will provide a powerful tool to select signal tracks and sharpen the turn-on curves.

\section{The phase-II tracker}

The current silicon strip tracker will age severely during phase-I operation and subsequently need replacement. The present tracker covers the pseudorapidity region up to $\mid$ eta $\mid=2.5$ and contains about ten barrel layers and endcap disks (see Figure 2-left), yielding a material thickness of about 1-1.5 radiation lengths. The new tracker (in Figure 2-right) is planned to be substantially lighter to reduce the photon conversion probability. Reducing sensor thickness and the number of layers are one contribution. At the same time its layout is improved, and L1 triggering capability will be added. The three inner barrel layers are made of systems in which "macro pixel modules" are paired with "strip modules" (shown in blue in Figure 2-right), designed to provide a measurement of the z position. The three outer layers (shown in red in Figure 2-right) are classical double strip modules. The tracker will remain placed inside the solenoid providing a 3.8T magnetic field, which is homogenous across the tracker and calorimeter volumes. As before, the iron return yoke will be immersed in the muon system.
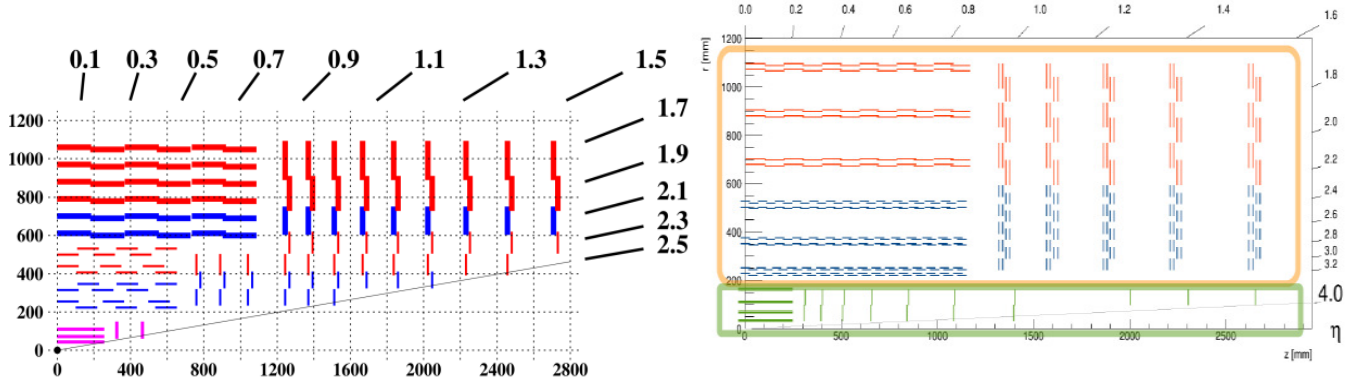

Figure 2. The phase-I (left) and phase-II (right) strip and pixel tracker geometry.

The innermost section of the tracker is currently equipped with a pixel vertex detector of $125 \mu \mathrm{m}^{2}$ pixel size. Also the pixel detector will have to be replaced. Simultaneously, it will be extended. The new vertex detector will have four (instead of three) pixel layers. Additional forward wheels will extend the coverage of the whole tracker up to $\mid$ eta $\mid=4$. This extension of forward instrumentation will have a big impact on the physics performance.

Owing to the reduced material budget, the resulting momentum resolution, depicted in Figure 3-left, is substantially improved to about $0.5 \%$ below $\mid$ eta $|\sim| 1$. Compared to the present performance (labelled as phase 1), in particular the resolution degradation in the range region between $1<\mid$ eta $\mid<1.5$ has disappeared. This region, corresponding to the barrel-endcap interface, at present holds a high density of services which is no longer present in the phase-II tracker with new powering and cooling schemes and cable routing. This reduces the rate of photon conversions which is an issue for example for precision measurements of the $\mathrm{H} \rightarrow \gamma \gamma$ channel.

The larger coverage in pseudorapidity improves the physics performance in many ways, very notably e.g. in the $\mathrm{H} \rightarrow \mathrm{ZZ} \rightarrow 4$ lepton channel. It also provides a good handle against jets arising from PU, which is especially important for vector-boson-fusion (VBF) channels characterized by two forward 
going jets originating at the primary vertex. Figure 3-right shows the ideal jet distribution in the absence of PU (black line) in comparison to scenarios with 140 PU events. Nearly 100 additional PU jets per event (in blue and green) would be wrongly reconstructed beyond eta 2 if no tracking hits would be present to identify the correct vertex. The additional instrumentation provides tracking hits in that region which allows to identify the vertex as a primary (signal) or PU-like vertex. The resulting distribution (in red) is very close to the ideal one.
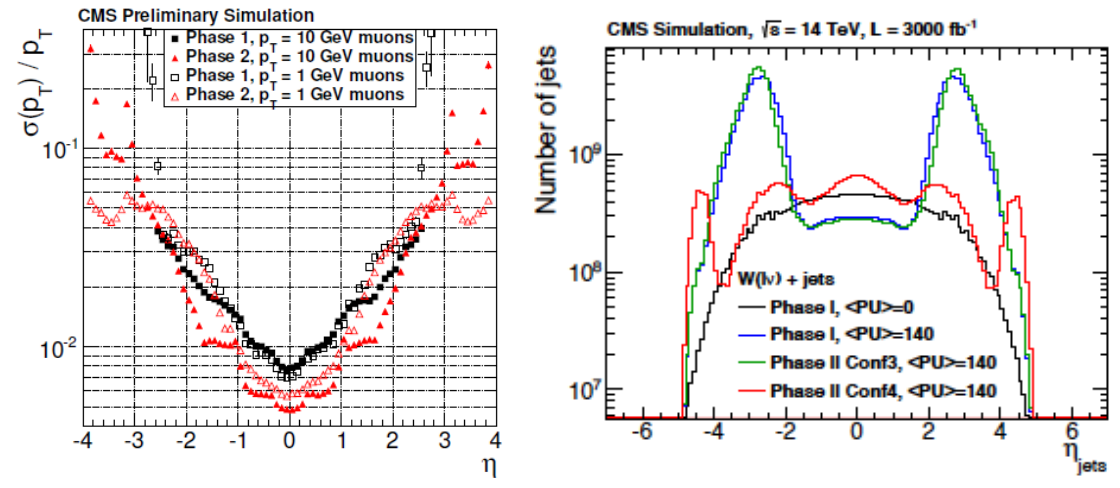

Figure 3. Physics performance of the upgraded tracker.

As a measure against PU, tracking information must be included in the level-1 (hardware-based) trigger which operates at $40 \mathrm{MHz}$. The objective of this "track trigger" is to reconstruct all tracks with $\mathrm{p}_{\mathrm{T}}>2 \mathrm{GeV}$ already at trigger level and to identify the primary vertices along the beam line with $\sim 1 \mathrm{~mm}$ precision. The concept exploits the strong magnetic field and the proximity of the tracking layer pairs as shown in Figure 2-right and in magnified view in Figure 4-left. Two suitable hits in neighboring sensors are combined to form a vector "stub" in the transverse plane. The deflection angle is a measure of the $\mathrm{p}_{\mathrm{T}}$. A striking benefit is the very sharp trigger turn-on as shown in black in Figure 4right in comparison to the run-1 configuration (in red). The latter shows a relatively slow turn-on, as it was the standard for decades of particle physics, thus necessitating an offline momentum cut significantly higher than the trigger threshold (e.g. $30 \mathrm{GeV}$ in the example, while the use of the track trigger would allow for a threshold $\sim 20 \mathrm{GeV}$ ). A crucial advantage is that trigger thresholds in general will be much lower since signal tracks can be separated distinctly from PU tracks through their vertex assignment. This is mandatory for B-physics with very soft muons, but also essential for SM and Higgs physics.
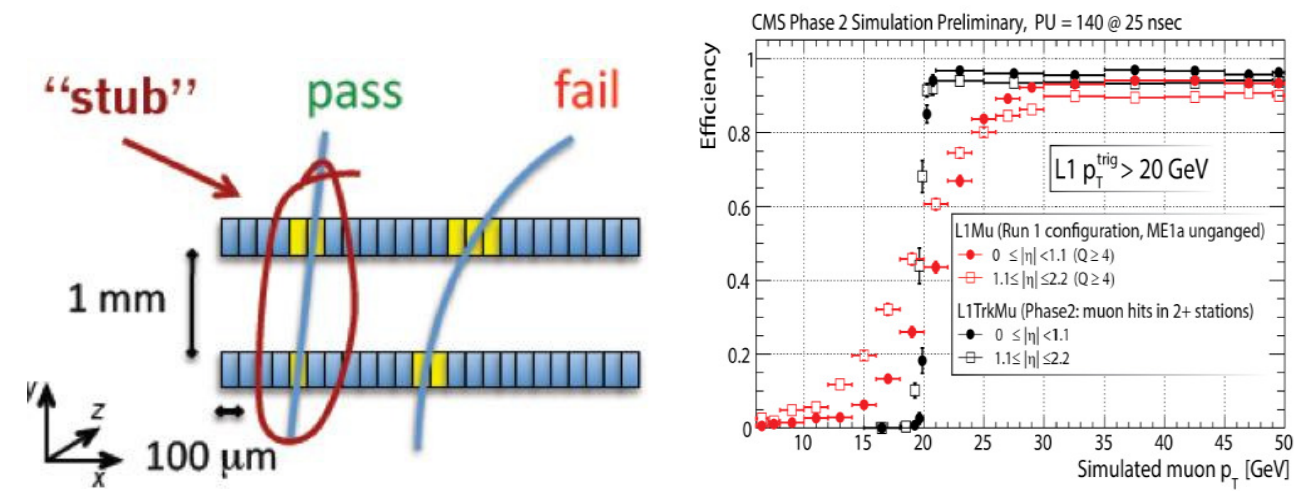

Figure 4. Track trigger conceptional design (left) and trigger performance (right). 


\section{The phase-Il calorimeters}

The present CMS calorimeters use $\mathrm{PbWO}_{4}$ crystals as active material in a homogeneous ECAL and a brass-scintillator sampling HCAL. The radiation dose is a strong function of eta, increasing by two to three orders of magnitude towards the forward regions. As a consequence, the current detector concept remains valid within the barrel region, where radiation damage is still viable and only the front-end electronics needs replacement to cope with the new trigger requirements. Figure 5-left shows the predicted degradation of the ECAL response, $\mathrm{S} / \mathrm{S}_{0}$ for $50 \mathrm{GeV}$ electrons as a function of eta for various integrated luminosities. After $3000 \mathrm{fb}^{-1}$, the ECAL barrel ( $\mid$ eta $\mid<1.48$ ) will have suffered less than a factor of two of light output reduction, while the forward regions will see a reduction by a factor of up to $10^{3}$, resulting in an unacceptable degradation of efficiency and energy resolution. The reduced output of scintillation light affects the energy resolution due to an amplification of the noise term along with a degradation of the stochastic term and a worsening of the constant term due to nonuniformity of the light collection along the crystal depth. The ECAL barrel crystals are read out with silicon photodiodes (APDs) with internal gain. Like all silicon devices they are sensitive to hadron radiation damage, with neutrons being the dominant source in this detector region. They create defects in the silicon bulk that increase the dark current. This leads to an increase of the electronics noise and a shift in the bias voltage, both resulting in a reduction of the quantum efficiency. One measure to reduce the electronics noise is a reduction of the operation temperature. The ECAL electronics will be replaced to meet the requirements of the phase-II trigger with increased latency up to $20 \mu \mathrm{s}$ and a bandwidth up to $1 \mathrm{MHz}$.
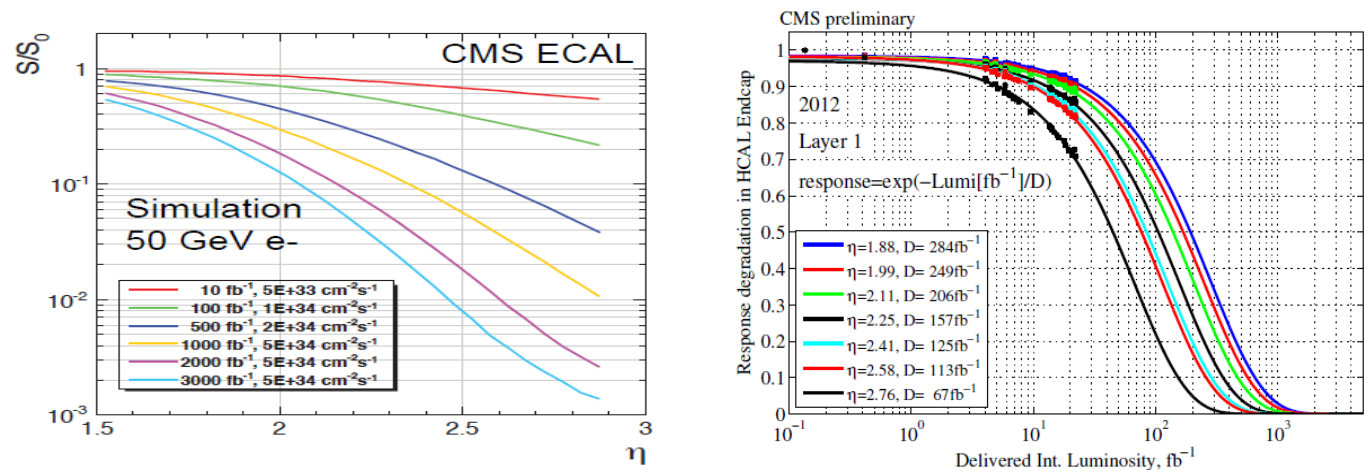

Figure 5. Response degradation for ECAL (left) and HCAL (right) due to aging.

Figure 5-right shows the corresponding response degradation for the brass-scintillator HCAL endcap, as a function of integrated luminosity and for specific eta values ranging from 1.88 to 2.76 . Measurements of the response up to now $\left(20 \mathrm{fb}^{-1}\right)$ are indicated as black dots in the figure; they provide the basis for the extrapolation to higher luminosity. In the forward region where the radiation levels are very high, as shown in Figure 1, the response is already degraded, and will approach zero even before reaching an integrated luminosity of $1000 \mathrm{fb}^{-1}$.

A replacement of both the electromagnetic and hadronic sections of the calorimeter endcap with radiation hard technologies is thus unavoidable.

After evaluation of the various technologies, two options have been selected for detailed studies to develop upgraded forward electromagnetic (EE) and hadronic (HE) endcap calorimeters. Option 1 depicted in Figure 6- largely maintains the present geometry with stand-alone electromagnetic and hadronic sections. The EE is designed as a sampling calorimeter without depth segmentation in Shashlik geometry made of very compact towers with a thickness of $\sim 25 \mathrm{X}_{0}$. Layers of absorber (2.5 $\mathrm{mm}$ of tungsten or brass) alternate with active detection layers of radiation-hard inorganic scintillating 
crystal tiles. The best performance is achieved with a material named LYSO (which stands for Cerium-doped Lutetium Yttrium Orthosilicate) with similar properties (e.g. density, energy resolution) as BGO but a shorter decay time (20 ns instead of $300 \mathrm{~ns}$, needed for HL operation) and about three times the $\mathrm{BGO}$ light yield while being radiation hard. LYSO (alternatively $\mathrm{CeF}_{3}$ ) provides good energy resolution and its small Moliere radius of about $14 \mathrm{~mm}$ allows for a fine granularity, which is necessary for efficient PU mitigation to match with the fine tracker granularity. The scintillation light is read out with four wavelength-shifting (WLS) quartz capillaries traversing the whole stack in either corner of the plates along with a monitoring fiber in the center. The fibers will be read out with solid-state photodetectors $(\mathrm{GaInP})$ which feature a better radiation hardness than silicon PMs due to their larger band gap. Such an EE is expected to provide an energy resolution of $\Delta \mathrm{E} / \mathrm{E}=10 \% / \sqrt{\mathrm{E}}$. The new $\mathrm{EE}$ will be more compact than the current endcap thanks to the shorter radiation length.

The shashlik EE option will be complemented with a hadronic section of similar segmented absorberscintillator geometry than the present one, but constructed with radiation hard components.
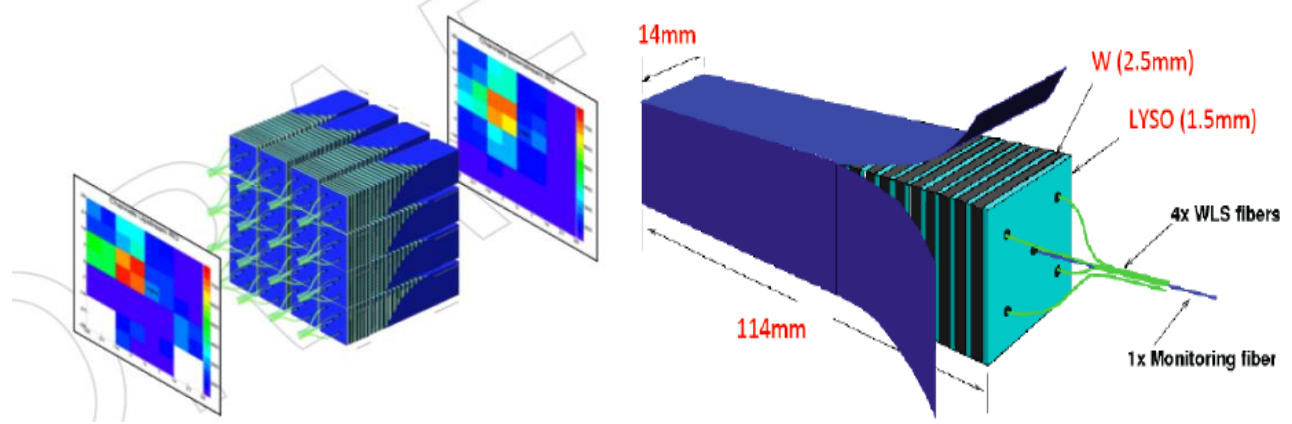

Figure 6. Option 1 for the upgrade of the forward calorimeters uses a shashlik geometry for the ECAL part.

The second option - shown in Figure 7 - is an integrated design as a high-granularity calorimeter (HGC) where EE and HE sections are implemented by varying segmentation and materials. All sections consist of alternating layers of dense absorber and silicon sensors as active material. The silicon sensors, having a typical cell size of $0.9 \mathrm{~cm}^{2}$ and a total surface of $420 \mathrm{~m}^{2}$, slide into thin gaps between the absorber which forms an alveolar mechanical structure (see Figure 7-left). EE and HE sections are designed according to their purpose. Moving outwards from the interaction region, the innermost electromagnetic section (E-HG) puts emphasis on radiation thickness with a depth of $\mathrm{X} / \mathrm{X}_{0} \sim 25$ (and $1 \lambda$ ) based on 31 layers and tungsten/lead as absorber, while the following HCAL section (H-HG) maximizes interaction length with 12 brass-scintillator sampling layers having a depth of of $3.5 \lambda$. A backing brass-scintillator calorimeter (B-HG) of $5.5 \lambda$ depth brings the total calorimeter depth to $10 \lambda$. 

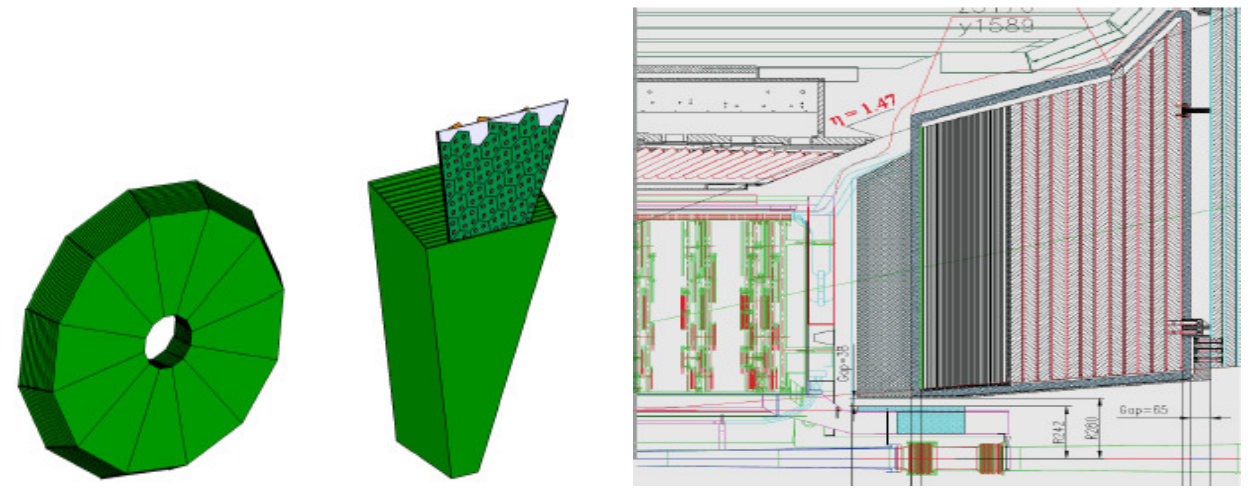

\section{Figure 7. Option 2 of the endcap calorimeter, foreseeing a high-granularity design based on silicon as an active material.}

The E-HG and H-HG will use planes of silicon sensors as active material, while the B-HG is well shielded by preceding detectors and can be constructed with either plastic scintillator or with GEM gaseous detectors. This structure should provide an improved jet energy measurement since the uninstrumented gap between EE and HE will be much smaller than in the present setup.

This design opens the possibility to extend the eta coverage to 4 and provides space for an additional muon tagging station behind the calorimeters in the very forward region (this detector is labelled as ME0 in Figure 8).

Such a calorimeter "visualizes" the energy flow through fine granularity based on its transverse longitudinal segmentation, and thus provides a high resolving power for single particles in very dense jets, as well as a good jet energy resolution exploiting the particle flow technique. It is expected to provide an energy resolution of $\Delta \mathrm{E} / \mathrm{E}=10 \% / \sqrt{\mathrm{E}}$, which is similar to that of the shashlik option.

\section{Upgrade of the muon system}

The CMS muon system is instrumented with two complementary detection technologies up to $\mid$ eta $\mid=1.6$ (see Figure 8). Drift chambers (DT) in the barrel and cathode strip chambers (labelled ME) in the endcaps provide precision measurements, complemented by resistive plate detectors (RPC, labelled RB and RE. At the time of construction of the original CMS experiment, it was not established that RPC detectors were capable of sustaining the challenging rates in the very forward region. For these reasons, no RPC modules are installed beyond |eta $\mid=1.6$.

This forward region is therefore one place to gain acceptance, as shown in Figure 9 for the decay of Higgs bosons to four muons. The conditions are very challenging: the background rates are very high, while the level of redundancy is the least. The B-field weakens towards the beam pipe since the field lines bend outwards at the edges. All these effects increase the probability to mis-measure the muon $\mathrm{p}_{\mathrm{T}}$ in the very forward region, resulting in fake triggers. For such muons with mis-measured momentum, increasing the threshold is not effective to control the muon trigger rate, thus these fake triggers are driving the muon trigger threshold to very high levels. Figure 9-left shows the trigger rate for the present detector as a function of the muon momentum (in blue). 


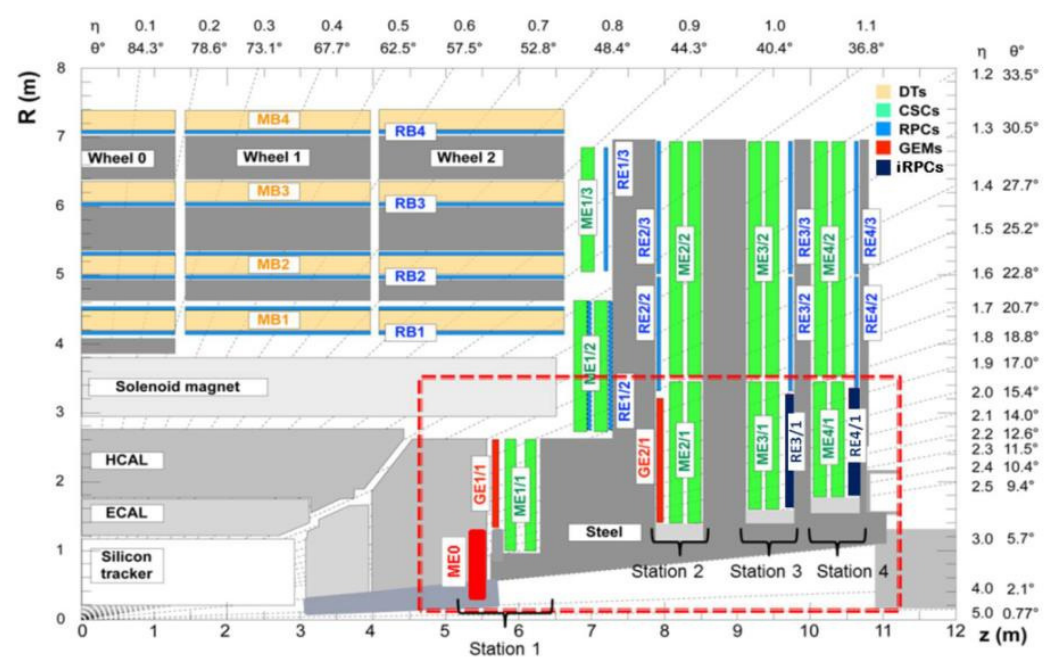

Figure 8. Upgrade of the muon system.

The missing redundancy will be re-established by installing high-rate capable detectors in the region $1.6<\mid$ eta $\mid<2.4$. For the first two stations (shown in red in Figure 8), triple-GEM detectors are foreseen. For stations three and four (blue in Fig.8) where the rates are lower due to the shielding between stations, the anticipated choice of detector technology will be improved, high-rate RPCs. For the latter, the Bakelite resistivity could be increased such that the charge extraction is significantly accelerated.

The magnetic field is still high at the very first muon endcap station (labelled ME1/1 in Figure 8) since it is positioned inside the solenoid. With a sufficient lever arm, the bending can be measured much more precisely. This is achieved by an additional GEM station (GE1/1) at a distance of about $30 \mathrm{~cm}$ from the CSC. Combining the measurements of both precision trackers allows measuring of the momentum with much greater precision, thus strongly reducing the level of mis-measurements. This effect is strongest in the first station where the B-field is high and diminishes for the other stations also due to multiple scattering. The resulting trigger rate - shown in Figure 9-left in purple - decreases much faster with momentum than with the present geometry, with the muon momentum correctly measured and hence following the expectation from SM processes.
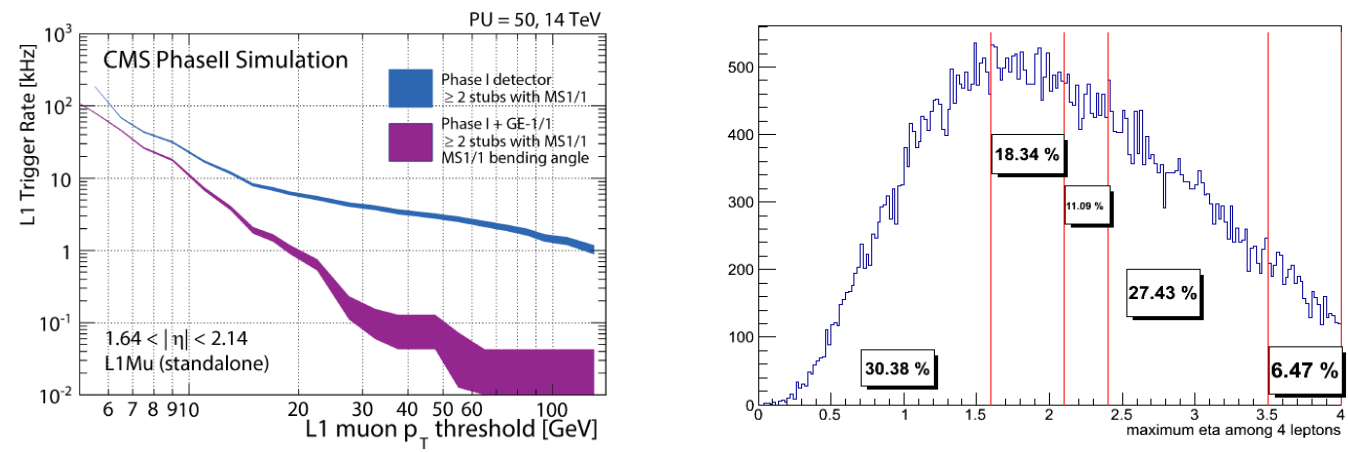

Figure 9. Left: Increasing the acceptance from eta $=2.4$ to eta $=4$ allows to collect an additional $\sim 35 \%$ of $\mathrm{H} \rightarrow \mathrm{ZZ} \rightarrow$ 4mu events. Right: At present, the $\mathrm{L1}$ muon trigger rate (in red) flattens out at high momenta due to mis-measured muons. This is improved by an additional muon station (GE1/1) at the innermost muon endcap station which improves the bending measurement in conjunction with the existing CSCs. 
Additional muon detectors will also be very beneficial for the performance of the offline muon reconstruction, as depicted in Figure 10. At present, beyond eta $=1.6$ only CSC detectors are installed. Although arranged as homogenous as possible, geometrical constraints (non-instrumented areas, services) yield lower reconstruction efficiencies in the regions around eta $=1.8$ and 2.05. Adding additional chambers will improve the efficiency significantly, as shown in Figure 10. This is even more true in case the CSC station 1 (labelled ME1/1) may malfunction for one reason or the other. In such cases, the newly installed, additional GE1/1 station will fully recover the missing hits in this very important region for physics.
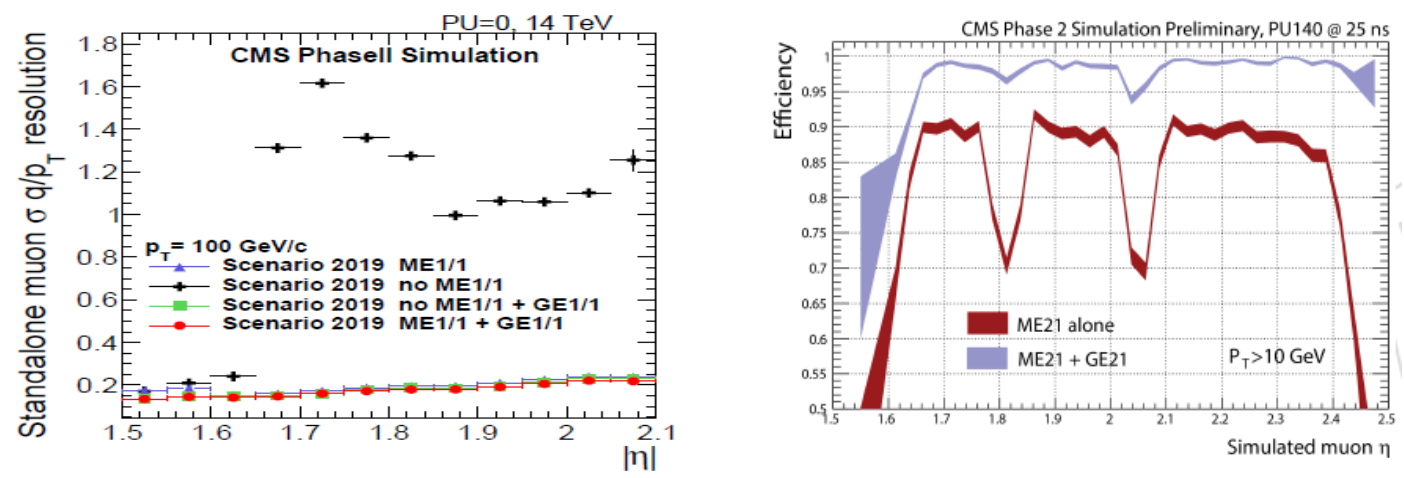

Figure 10. Benefits of the additional detectors in the muon system for offline muon reconstruction.

\section{Conclusions and summary}

Following a substantial upgrade of the LHC accelerator in long-shutdown 3, around 2025 LHC phase-II is expected to operate at about five times higher luminosity, then exceeding the original design parameters of the LHC detectors in terms of luminosity and overall detector lifetime. As a consequence of this high luminosity, about 140 additional pile-up events are to be identified to extract rare physics signals from the large background. To achieve the goal to maintain the present detector performance several upgrades are necessary. Readout and trigger systems are being re-designed using recent technology developments. The high particle rates will accelerate detector aging and require replacement of the tracker and forward calorimeters. The new tracker geometry is optimized for trigger and physics performance in a high rate environment. Two designs are under study for the forward calorimetry, a shashlik geometry based on scintillation light and a high-granularity option exploiting silicon as an active detection element. The muon system will be extended in the very forward region with additional high-rate gaseous detectors (GEM and RPC). 


\section{References}

1. CMS Collaboration, "The CMS experiment at the CERN LHC", JINST 3 (2008), no. S08004, doi:10.1088/1748-0221/3/08/S08004.

2. CMS Collaboration, "Technical proposal for the CMS phase-II upgrade", document in preparation 